\title{
Impaired mitochondrial oxidative phosphorylation and supercomplex assembly in rectus abdominis muscle of diabetic obese individuals
}

\author{
Ghadi Antoun $^{1} \cdot$ Fiona McMurray ${ }^{1}$ - A. Brianne Thrush ${ }^{1}$ - David A. Patten ${ }^{2}$. \\ Alyssa C. Peixoto ${ }^{1} \cdot$ Ruth S. Slack ${ }^{2} \cdot$ Ruth McPherson $^{3} \cdot$ Robert Dent $^{4}$ • \\ Mary-Ellen Harper ${ }^{1}$
}

Received: 9 July 2015 / Accepted: 1 September 2015 /Published online: 24 September 2015

(C) Springer-Verlag Berlin Heidelberg 2015

\begin{abstract}
Aims/hypothesis Skeletal muscle mitochondrial dysfunction has been documented in patients with type 2 diabetes mellitus; however, specific respiratory defects and their mechanisms are poorly understood. The aim of the current study was to examine oxidative phosphorylation and electron transport chain (ETC) supercomplex assembly in rectus abdominis muscles of 10 obese diabetic and 10 obese non-diabetic individuals.

Methods Twenty obese women undergoing Roux-en-Y gastric bypass surgery were recruited for this study. Muscle samples were obtained intraoperatively and subdivided for multiple analyses, including high-resolution respirometry and assessment of supercomplex assembly. Clinical data obtained from referring physicians were correlated with laboratory findings.

Results Participants in both groups were of a similar age, weight and BMI. Mitochondrial respiration rates were
\end{abstract}

Fiona McMurray and A. Brianne Thrush contributed equally to this work.

Electronic supplementary material The online version of this article (doi:10.1007/s00125-015-3772-8) contains peer-reviewed but unedited supplementary material, which is available to authorised users.

Mary-Ellen Harper

mharper@uottawa.ca

1 Department of Biochemistry, Microbiology and Immunology, Faculty of Medicine, University of Ottawa, 451 Smyth Rd, Ottawa, ON, Canada K1H 8M5

2 Department of Cellular and Molecular Medicine, Faculty of Medicine, University of Ottawa, Ottawa, ON, Canada

3 Atherogenomics Laboratory, University of Ottawa Heart Institute, Ottawa, ON, Canada

4 Ottawa Hospital Weight Management Clinic, The Ottawa Hospital, Ottawa, ON, Canada markedly reduced in diabetic vs non-diabetic patients. This defect was observed during maximal ADP-stimulated respiration in the presence of complex I-linked substrates and complex I- and II-linked substrates, and during maximal uncoupled respiration. There were no differences in fatty acid (octanoyl carnitine) supported respiration, leak respiration or isolated activity of cytochrome c oxidase. Intriguingly, significant correlations were found between glycated haemoglobin $\left(\mathrm{HbA}_{1 \mathrm{c}}\right)$ levels and maximal respiration or respiration supported by complex I, complex I and II or fatty acid. In the muscle of diabetic patients, blue native gel electrophoresis revealed a striking decrease in complex I, III and IV containing ETC supercomplexes.

Conclusions/interpretation These findings support the hypothesis that ETC supercomplex assembly may be an important underlying mechanism of muscle mitochondrial dysfunction in type 2 diabetes mellitus.

Keywords Bioenergetics · Electron transport chain . Mitochondria · Obesity $\cdot$ Skeletal muscle $\cdot$ Type 2 diabetes mellitus
Abbreviations
COX Cytochrome c oxidase
ETC Electron transport chain
ETF Electron transfer flavoprotein

\section{Introduction}

Type 2 diabetes mellitus is characterised by insulin resistance in a variety of tissues. Skeletal muscle is normally accountable for $80-90 \%$ of postprandial glucose uptake, but it becomes a 
major site of insulin resistance during the development of type 2 diabetes mellitus. Mitochondrial dysfunction in muscle has been the focus of many studies, some of which have identified altered mitochondrial morphology [1] and downregulation of genes involved in oxidative phosphorylation (e.g. peroxisome proliferator-activated receptor gamma coactivator 1-alpha $[\mathrm{PGC} 1 \alpha]$-responsive genes) [2]. Metabolic inflexibility, or the inability to optimally switch between carbohydrate and fatty acid fuel sources [3], and impairments in mitochondrial electron transport chain (ETC) components $[1,4]$ have also been described in the muscle of patients with type 2 diabetes mellitus. Recently, DeLany et al demonstrated dysfunctional mitochondrial respiration before the appearance of any clinical characteristics of type 2 diabetes mellitus in a population of African-American women [5]. Despite these clear associations between mitochondrial dysfunction and the risk of developing type 2 diabetes mellitus, the underlying mechanisms in human skeletal muscle are poorly understood.

Here we report impaired skeletal muscle mitochondrial energetics in individuals with type 2 diabetes mellitus and show that this is significantly correlated with circulating levels of glycated haemoglobin $\left(\mathrm{HbA}_{1 \mathrm{c}}\right)$. Moreover, we demonstrate that there are corresponding decreases in the formation of mitochondrial ETC supercomplexes. Since ETC supercomplexes can regulate mitochondrial respiration [6], our findings suggest these may represent important mechanistic players of disordered skeletal muscle metabolism in the aetiology of type 2 diabetes mellitus.

\section{Methods}

Twenty female patients from the Ottawa Hospital Weight Management Clinic were recruited after being confirmed as candidates for bariatric surgery. The study was approved by the Ottawa Hospital Ethics Board and written informed consent was obtained from each patient prior to study participation. Patients were included in this study based on their availability, willingness to participate and eligibility for gastric bypass surgery. They were recruited randomly as part of an ongoing sample collection of consenting individuals referred for bariatric surgery. No a priori selection was made based on medications taken by patients in each group. There were no differences between groups in the use of other commonly prescribed agents for BP, depression, gastroesophageal reflux and dyslipidemia. Diabetes medications included metformin, sulfonylureas (e.g. gliclazide), dipeptidyl peptidase 4 (DPP-4) inhibitors (e.g. sitagliptin), glucagon-like peptide-1 (GLP-1) agonists (e.g. liraglutide), sodium/glucose cotransporter 2 (SGLT2) inhibitors (e.g. canagliflozin) and various insulin derivatives (e.g. detemir [B29Lys( $\varepsilon$-tetradecanoyl),desB30 human insulin], glargine [A21 Gly,B31 Arg,B32Arg human insulin] and aspart [B28Asp human insulin] insulins). All medications were discontinued on the day of surgery and blood glucose levels were measured. Diabetic patients received intravenous dextrose and/or insulin as necessary.

Exclusion criteria included symptomatic coronary artery disease, inflammatory bowel disease (Crohn's) and oesophageal varices. Patients were also excluded if they were aged over 65 years or if their $\mathrm{HbA}_{1 \mathrm{c}}$ levels were $>8.0 \%(64 \mathrm{mmol} / \mathrm{mol})$. Prior to surgery, all patients underwent routine preparations for Roux-en-Y gastric bypass including OPTIFAST ${ }^{\circledR} 900$ meal replacement for 3 weeks. Patients fasted for at least $8 \mathrm{~h}$ prior to the surgery and sample collection. No muscle-conditioning programme was prescribed to patients and exercise was limited, given their very high BMIs $\left(\sim 50 \mathrm{~kg} / \mathrm{m}^{2}\right)$.

Rectus abdominis samples were obtained during Roux-en-Y gastric bypass surgery. Samples were divided for multiple analyses including high-resolution respirometry, assessment of ATP synthase and ETC supercomplex assembly by blue native polyacrylamide gel electrophoresis and measurement of individual components by traditional Western blotting. Analysis of mitochondrial function by high-resolution respirometry was performed using the Oxygraph-2k modular system for highresolution respirometry (OROBOROS Instruments, Innsbruck, Austria), as previously described [7]. For additional details on methods, please refer to electronic supplementary material (ESM) Methods.

Statistical analysis GraphPad Prism 6 (La Jolla, CA, USA) was used to perform Unpaired Student's $t$ tests and regression analyses, and data are presented as means \pm SEM.

\section{Results}

Baseline characteristics of all participants are outlined in ESM Table 1. There were no differences in mean age, weight, BMI and percentage body fat of participants in the obese non-diabetic and obese diabetic groups. All patients had a BMI $>40 \mathrm{~kg} / \mathrm{m}^{2}$ and $\mathrm{HbA}_{1 \mathrm{c}}$ values were significantly higher in diabetic patients $(5.5 \pm 0.1 \%[36.8 \pm 1.6 \mathrm{mmol} / \mathrm{mol}]$ vs $7.3 \pm$ $0.2 \%[55.7 \pm 2.3 \mathrm{mmol} / \mathrm{mol}], p<0.001)$.

In order to assess differences in mitochondrial energetics, high-resolution respirometry was performed on permeabilised rectus abdominis muscle fibres. Respiration was decreased in muscle samples from diabetic patients (Fig. 1). This was observed across a variety of phosphorylating respiratory states (state 3), including in the presence of complex I linked substrates $\left(\mathrm{P}_{\mathrm{CI}} ; 20.7 \pm 2.5 \mathrm{vs} 11.8 \pm 2.2 \mathrm{pmol} /[\mathrm{s} \times \mathrm{mg}\right.$ wet weight], $p=0.02$; Fig. 1a) and complex I and II linked substrates $\left(\mathrm{P}_{\mathrm{CI}+\mathrm{CII}} ; 50.4 \pm 4.1\right.$ vs $35.0 \pm 2.2 \mathrm{pmol} /[\mathrm{s} \times \mathrm{mg}$ wet weight] , $p=0.004$; Fig. 1b). Maximal respiration in the presence of the uncoupler carbonylcyanide- $p$-trifluoromethoxyphenylhydrazone (FCCP) was also significantly lower in diabetic patients $\left(\mathrm{U}_{C} ; 57.1 \pm 5.3\right.$ vs $38.4 \pm 2.7 \mathrm{pmol} /[\mathrm{s} \times \mathrm{mg}$ wet weight], 

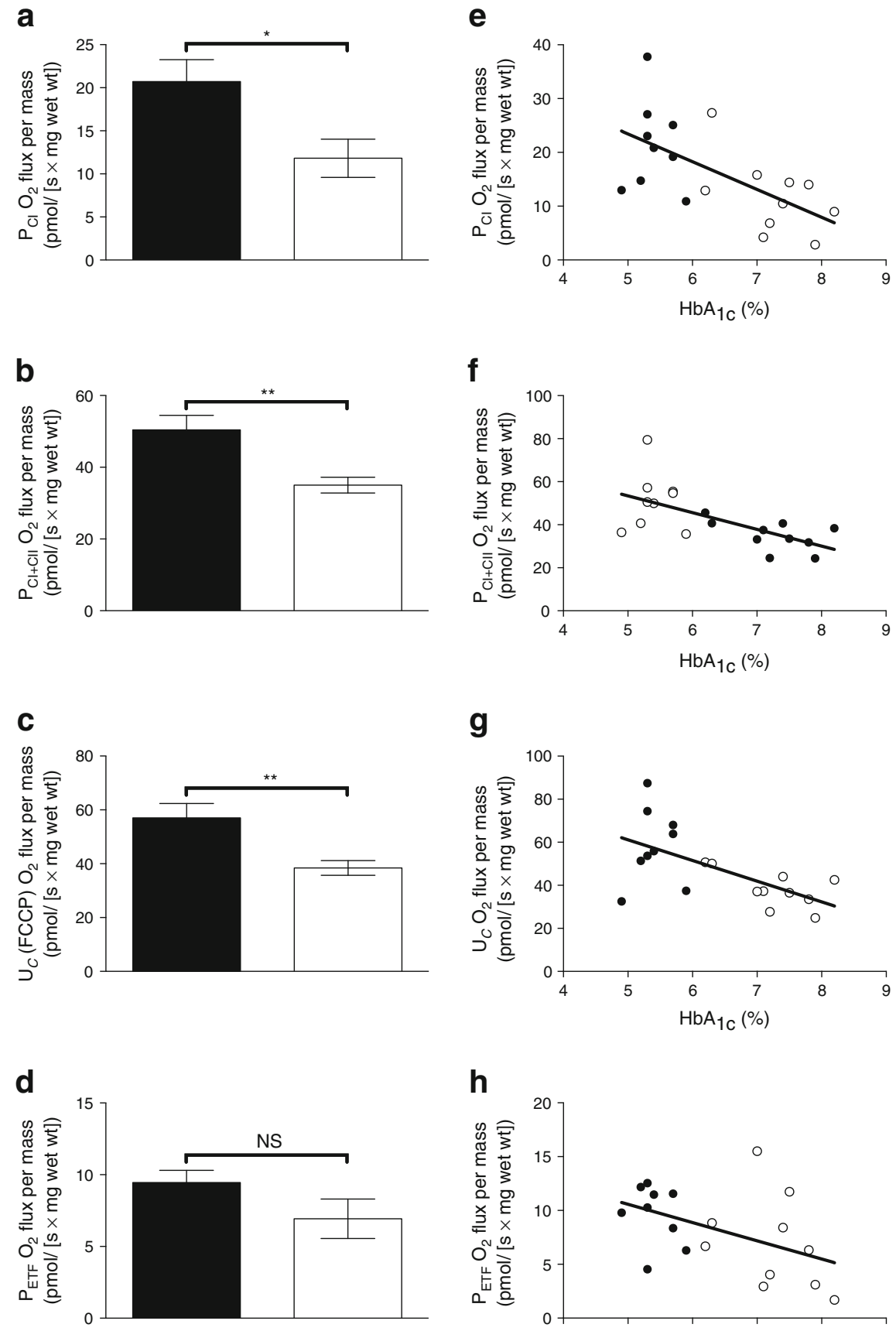

h

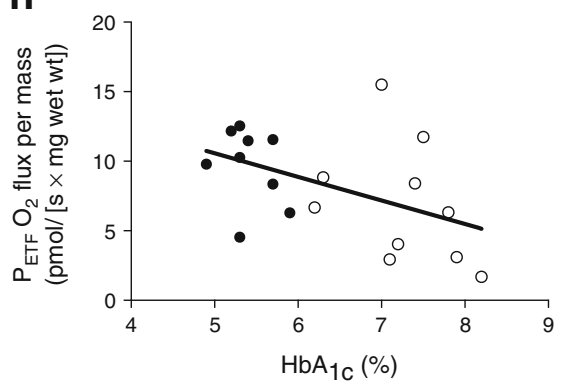

Fig. 1 Mitochondrial energetics are altered in permeabilised rectus abdominis muscle fibres from obese diabetic women compared with obese non-diabetic women, and correlate significantly with $\mathrm{HbA}_{1 \mathrm{c}}$ levels. Data are shown for state 3 respiratory capacity through (a, e) complex I $\left(\mathrm{P}_{\mathrm{CI}}\right),(\mathbf{b}, \mathbf{f})$ maximal oxidative phosphorylation capacity $\left(\mathrm{P}_{\mathrm{CI}+\mathrm{CII}}\right),(\mathbf{c}, \mathbf{g})$ maximal uncoupled respiration in the presence of the chemical protonophore carbonylcyanide- $p$-trifluoromethoxyphenylhydrazone $\left(\mathrm{U}_{C}\right)$, and $(\mathbf{d}, \mathbf{h})$ maximal electron flow through electron-transferring

$p=0.006$; Fig. 1c). There was a trend toward lower fatty acid supported respiration in the presence of electron transfer flavoprotein (ETF) linked substrates, such as octanoyl carnitine

flavoprotein and fatty acid oxidative capacity $\left(\mathrm{P}_{\mathrm{ETF}}\right)$. (a-d) All values are mean $\pm \mathrm{SEM}, n=10$ non-diabetic patients (black bars) and $n=10$ diabetic patients (white bars). ${ }^{*} p<0.05,{ }^{* *} p<0.01$ for the unpaired Student's $t$ test; (e) $p=0.0042, R^{2}=0.3910$; (f) $p=0.0034, R^{2}=0.4045$; (g) $p=0.0044, R^{2}=0.3886$; (h) $p=0.0405, R^{2}=0.2243$. (e-h) All data were obtained by linear regression analyses, $n=9$ non-diabetic patients (black squares) and $n=10$ diabetic patients (white points). To convert values for $\mathrm{HbA}_{1 \mathrm{c}}$ in \% into $\mathrm{mmol} / \mathrm{mol}$, subtract 2.15 and multiply by 10.929

( $\mathrm{P}_{\mathrm{ETF}}$; Fig. 1d). No differences were observed in nonphosphorylating leak respiration in the absence of ADP $\left(\mathrm{L}_{\mathrm{N}}\right.$; ESM Fig. 1a) or in the presence of oligomycin, an 
ATP synthase (complex V) inhibitor ( $\mathrm{L}_{\text {Omy }}$; ESM Fig. 1b). Further normalisation of data to the activity of cytochrome c oxidase, as assessed by $N, N, N^{\prime}, N^{\prime}-$ tetramethyl- $p$-phenylenediamine (TMPD; Tm; ESM Fig. 1c), did not alter the results (ESM Fig. 2).

\section{a}
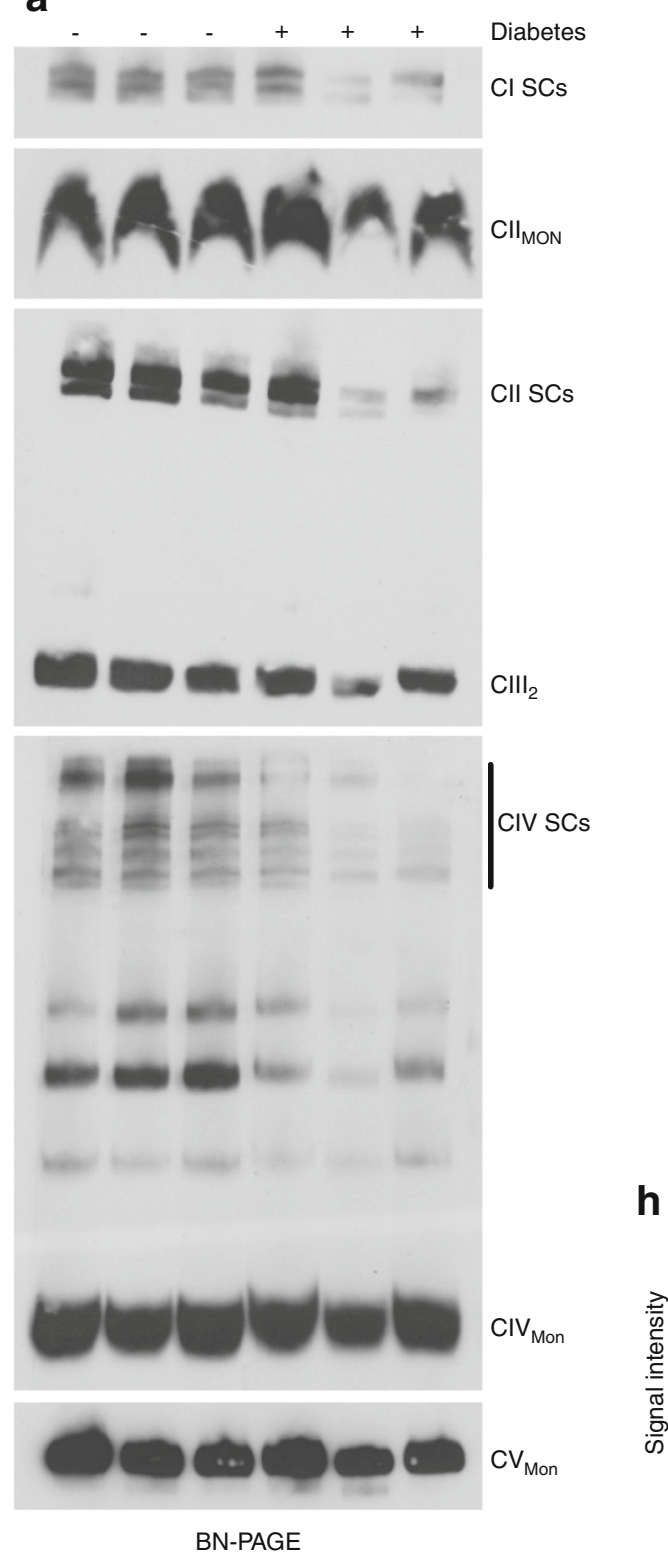

Fig. 2 Supercomplex assembly is altered in rectus abdominis muscle samples from obese diabetic women compared with obese non-diabetic women. (a) Representative blue native polyacrylamide gel (BN-PAGE) blot of the indicated respiratory complexes (CI-V), supercomplexes (SCs), and monomers (Mon) using anti-NADH dehydrogenase [ubiquinone] 1 alpha subcomplex subunit 9, mitochondrial (NDUFA9; complex I), anti-flavoprotein (complex II), anti-ubiquinol-cytochrome c reductase core protein II (UQCRC2; complex III), anti-complex IV subunit I (complex IV) and anti-ATP synthase subunit alpha, mitochondrial (ATP5a; complex V) antibodies. (b-f) Quantification of expression of the indicated respiratory supercomplex normalised to complex II monomer
A significant inverse correlation was found between circulating levels of $\mathrm{HbA}_{1 \mathrm{c}}$ and respiration through complex I $\left(\mathrm{P}_{\mathrm{CI}}\right.$; Fig. 1e), complex I and II ( $\mathrm{P}_{\mathrm{CI}+\mathrm{CII}}$; Fig. 1f), maximal uncoupled respiration $\left(\mathrm{U}_{C}\right.$; Fig. $\left.1 \mathrm{~g}\right)$ and fatty acid oxidative capacity $\left(\mathrm{P}_{\mathrm{ETF}}\right.$; Fig. $\left.1 \mathrm{~h}\right) . \mathrm{HbA}_{1 \mathrm{c}}$ was also negatively associated
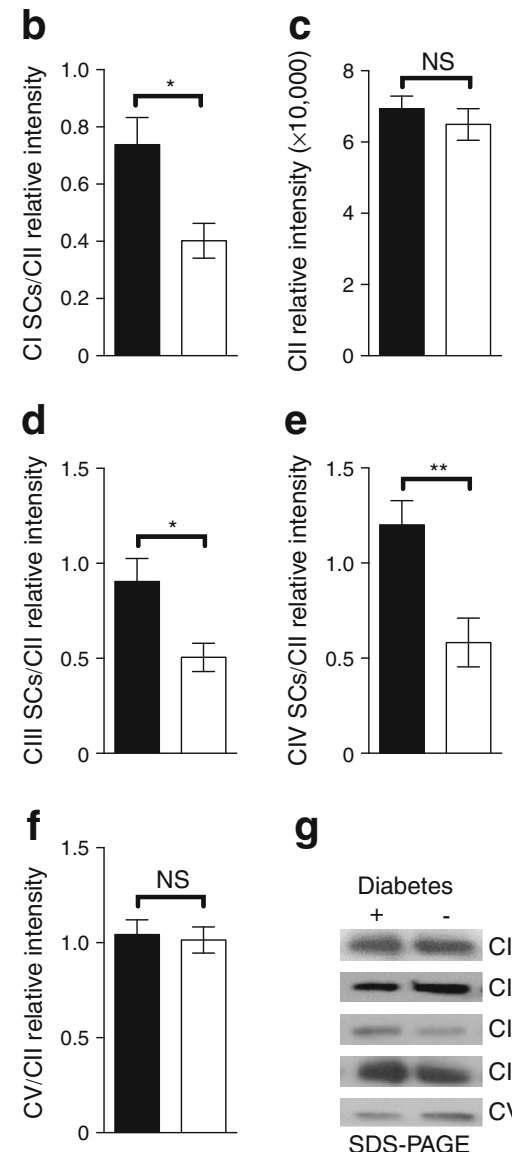

g
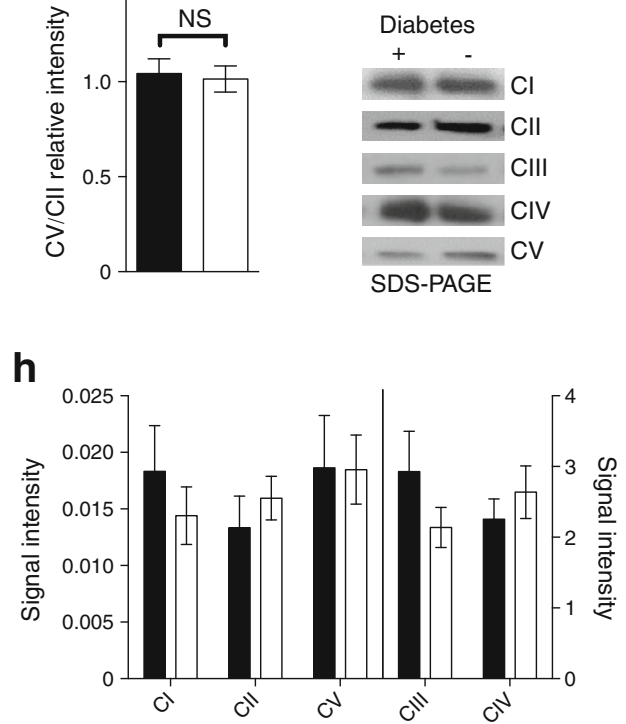

levels. Complex II monomer levels in (c) were normalised to Ponceau S staining. $n=6$ non-diabetic patients (black bars) and $n=7$ diabetic patients (white bars). (g) Representative western blot of the indicated respiratory complexes using anti-NDUFA9 (complex I), anti-flavoprotein (complex II), anti-complex III subunit core 1 (complex III), anti-complex IV subunit I (complex IV) and anti-ATP5a (complex V) antibodies. (h) Quantification of the indicated respiratory complexes (total levels). $n=5$ nondiabetic patients (black bars) and $n=7$ diabetic patients (white bars). All values are the mean \pm SEM. ${ }^{*} p<0.05,{ }^{*} p<0.01$ for the unpaired Student's $t$ test 
with adenylate-free leak respiration $\left(\mathrm{L}_{\mathrm{N}}\right.$; ESM Fig. 1d; $p=0.051)$. In contrast, no correlation was observed between $\mathrm{HbA}_{1 \mathrm{c}}$ and oligomycin-induced leak respiration ( $\mathrm{L}_{\text {Omy }}$; ESM Fig. 1e) and mitochondrial content (Tm; ESM Fig. 1f). When data were analysed in the same manner, but using data from the diabetic women only, maximal uncoupled respiration was the only respiration type to reach near significance $(p=0.0674)$.

In order to further investigate the causes of reductions in mitochondrial respiration, higher order ETC supercomplex assembly was assessed (Fig. 2). There was less supercomplex formation in the muscle of diabetic patients (Fig. 2a). This was consistently observed when the subunits of complex I (Fig. 2b), complex III (Fig. 2d) and complex IV (Fig. 2e) were analysed separately. ATP synthase and complex II do not participate in mammalian ETC supercomplex formation, and were similar in both patient groups (Fig. 2c, f). For this reason, all protein levels were normalised to the complex II monomer level. Additionally, no differences were observed in the absolute amount of any complex as assessed by reducing SDSPAGE blots (Fig. 2g, h). This suggests that reductions in supercomplex assembly do not correlate with changes in the total levels of ETC complexes.

\section{Discussion}

Previous reports have examined mitochondrial dysfunction in obese type 2 diabetes mellitus patients [1, 4, 8, 9]. Our results confirm the presence of mitochondrial dysfunction and significantly extend previous findings by characterising the various states of mitochondrial energetics in intact permeabilised human muscle fibres and assembly of ETC supercomplexes. This constitutes the first report of altered mitochondrial supercomplex assembly in type 2 diabetes mellitus and of mitochondrial energetics in permeabilised rectus abdominis muscle fibres. We also identify novel correlations between human muscle mitochondrial energetics (including fatty acid metabolism) and circulating $\mathrm{HbA}_{1 \mathrm{c}}$ levels.

Previous reports linking dysfunctional mitochondrial energetics with type 2 diabetes mellitus are limited, and many have been based upon analyses of the maximum velocity $\left(V_{\max }\right)$ enzyme activities of individual ETC proteins, as measured in frozen muscle samples $[1,4,9]$ or permeabilised mitochondria [8]. Our ex vivo methods in which mitochondrial energetics are analysed in permeabilised muscle fibres are arguably the least disruptive to the in vivo mitochondrial network. Overall, the results presented herein agree with previous findings and demonstrate substantial reductions in the different respiratory states, including fatty acid supported and maximal respiration. Previous studies have been conducted using vastus lateralis muscle samples from obese individuals with considerably lower BMIs $\left(<35 \mathrm{~kg} / \mathrm{m}^{2}\right)$, most often in men [8-10], whereas the current study focuses on differences observed in the rectus abdominis muscle of women with a much higher BMI (ESM Table 1). These differences in studied muscle groups and patient populations may partly explain why the findings presented herein contradict the findings of Boushel et al [10]. A further explanation for these discrepancies could be that the data presented here are normalised against COX activity within the same sample, whereas Boushel et al [10] normalised data to citrate synthase activity measured in a separate sample of whole muscle lysate. Taken together, these results support the idea that deficiencies in muscle mitochondrial respiration are global, surpassing the boundaries of the specific muscle group tested and the sex of the affected individual.

As mitochondrial content can substantially impact respiratory capacity, it was assessed by quantification of protein components of individual respiratory complexes (Fig. 2g). Consistent with previous work $[8,10]$, we observed no differences in mitochondrial content of muscle between diabetic and nondiabetic patients. Additionally, functional analysis of complex IV (cytochrome c oxidase) maximal activity (ESM Fig. 1c), which is sometimes used as a proxy measure of mitochondrial content, revealed no differences between the diabetic and nondiabetic groups.

While investigating the mechanism of this mitochondrial dysfunction, we discovered pronounced reductions in mitochondrial supercomplex assembly. Others have demonstrated that reductions in mitochondrial function precede the development of obesity and type 2 diabetes mellitus in a high-risk population [5]. These findings were significant across a variety of respiratory states, leading us to hypothesise that supercomplex formation may be inherently altered in highrisk populations. The association of ETC proteins into supercomplexes has been proposed to promote electron flow through the ETC, which in turn supports augmented respiration rates [6].

Supercomplex formation is an as yet poorly understood process that is affected by factors such as cristae structure, membrane integrity and levels of specific proteins including ubiquinol-cytochrome-c reductase complex assembly factor 3 (C11ORF83) and cytochrome c oxidase subunit 7A-related protein, mitochondrial (COX7RP). An additional protein affecting cristae structure and ETC supercomplex assembly is mitofilin, a component of the mitochondrial contact site and cristae organising system (MICOS). Interestingly, overexpression of mitofilin was shown to rescue deficiencies in respiration in a mouse model of type 1 diabetes mellitus, although rescue of ETC supercomplex assembly was not investigated [11]. Furthermore, the cardiolipin content of the mitochondrial inner membrane has been shown to affect mitochondrial function and supercomplex formation, and may be involved in the pathophysiology of diabetes [4]. Future studies are required to determine the causes of the supercomplex dysfunction observed in this study. 
In conclusion, we report extensive mitochondrial dysfunction in rectus abdominis muscle of obese diabetic women. Mitochondrial respiration negatively correlated with circulating levels of $\mathrm{HbA}_{1 \mathrm{c}}$. Furthermore, dysfunctional mitochondrial energetics in muscle of diabetic patients were linked to reductions in the assembly of mitochondrial supercomplexes, suggesting that this is an important mechanism for development of the disease. Future studies will be required to delineate approaches that could reverse this dysfunction.

Acknowledgements We would like to thank the patients for their generous participation in this study. We would also like to thank H. Doelle, University of Ottawa Heart Institute, and the bariatric surgery team of the Ottawa Hospital, specifically J. Mamazza, J.-D. Yelle, I. Raiche and A. Neville, for their invaluable contribution to patient recruitment and sample collection.

Funding The work was funded by the Canadian Institutes of Health Research (CIHR) (grant nos MOP57810 and MOP136936). GA was supported by the Vanier Canada Graduate Scholarship Program. FM was supported by a University of Ottawa Vision 2020 Postdoctoral Fellowship. ABT was supported by a CIHR Postdoctoral Fellowship. DAP was supported by a Heart and Stroke Foundation Doctoral Research Award.

Contribution statement All authors contributed to the conception and design of the study. GA, FM, ABT and RD acquired the clinical data; GA, FM, ABT, DAP and ACP generated respirometry data and performed all laboratory work; data were analysed and interpreted by GA, FM, ABT, DAP, ACP, RSS, RM and M-EH. The manuscript was drafted by GA, FM, ABT, DAP, ACP, RSS and M-EH and critical revisions were performed by all authors. All authors have approved the final version. GA and M-EH are the guarantors of this work.

Duality of interest statement The authors declare that there is no duality of interest associated with this manuscript.

\section{References}

1. Kelley DE, He J, Menshikova EV, Ritov VB (2002) Dysfunction of mitochondria in human skeletal muscle in type 2 diabetes. Diabetes 51:2944-2950

2. Mootha VK, Lindgren CM, Eriksson KF et al (2003) PGC-1alpharesponsive genes involved in oxidative phosphorylation are coordinately downregulated in human diabetes. Nat Genet 34:267-273

3. Kelley DE (2005) Skeletal muscle fat oxidation: timing and flexibility are everything. J Clin Invest 115:1699-1702

4. Ritov VB, Menshikova EV, Azuma K et al (2010) Deficiency of electron transport chain in human skeletal muscle mitochondria in type 2 diabetes mellitus and obesity. Am J Physiol Endocrinol Metab 298:E49-E58

5. DeLany JP, Dube JJ, Standley RA et al (2014) Racial differences in peripheral insulin sensitivity and mitochondrial capacity in the absence of obesity. J Clin Endocrinol Metab 99:4307-4314

6. Lapuente-Brun E, Moreno-Loshuertos R, Acin-Perez R et al (2013) Supercomplex assembly determines electron flux in the mitochondrial electron transport chain. Science 340:1567-1570

7. Pesta D, Gnaiger E (2012) High-resolution respirometry: OXPHOS protocols for human cells and permeabilized fibres from small biopsies of human muscle. Methods Mol Biol 810:25-58

8. Mogensen M, Sahlin K, Fernstrom M et al (2007) Mitochondrial respiration is decreased in skeletal muscle of patients with type 2 diabetes. Diabetes 56:1592-1599

9. Ritov VB, Menshikova EV, He J, Ferrell RE, Goodpaster BH, Kelley DE (2005) Deficiency of subsarcolemmal mitochondria in obesity and type 2 diabetes. Diabetes 54:8-14

10. Boushel R, Gnaiger E, Schjerling P, Skovbro M, Kraunsoe R, Dela F (2007) Patients with type 2 diabetes have normal mitochondrial function in skeletal muscle. Diabetologia 50:790-796

11. Thapa D, Nichols CE, Lewis SE et al (2015) Transgenic overexpression of mitofilin attenuates diabetes mellitus-associated cardiac and mitochondria dysfunction. J Mol Cell Cardiol 79:212-223 